INDO GLOBAL JOURNAL OF

PHARMACEUTICAL SCIENCES

ISSN 2249- 1023

\title{
A Nanotechnological Approach to Improve Oral Antidiabetic Efficacy of Phytochemicals Extracted from Tinospora Cordifolia: In-Vivo and Ex-Vivo Studies
}

\author{
Arun Kumar, Maneesh Jaiswal * \\ Department of Pharmacy, Jaypee University of Information Technology, Waknaghat, Solan-173215, H.P., India \\ Address for Correspondence: Maneesh Jaiswal; jaiswal.maneesh@gmail.com , maneesh.jaiswal@ juit.ac.in
}

\begin{abstract}
Present study demonstrates the designing and development of nanoemulsion based drug delivery system of phytochemicals from Tinospora Cordifolia to improve their oral antidiabetic efficacy. Nanoformulations were characterized and extensively investigated for size, morphology, and drug release profile as well as ex-vivo permeation studies. Nanodroplet size and zeta potential of nanoformulation were optimized and were found within the range of $60 \mathrm{~nm}$ to $180 \mathrm{~nm}$. Raw extract and nanoemulsion loaded with drug releases $79.4 \pm 1.5 \%$ and $80.5 \pm 0.9$ after $12 \mathrm{~h}$ and $24 \mathrm{~h}$ respectively. Approximately 6.4 fold enhancement in the value of permeability coefficient of $T$. cordifolia was noticed upon formulating it as nanoemulsion. Nanoformulations were found to improve the intestinal membrane permeability, greater biological stability and sustained release for prolonged period of time and have showed excellent in-vitro antioxidant efficacy in compare to control. These formulations were proved to be good candidates for oral drug delivery and can further investigate for in-vivo animal experiments. () 2014 iGlobal Research and Publishing Foundation. All rights reserved.
\end{abstract}

Conference Proceedings: International Conference on Life Sciences, Informatics, Food and Environment; August 29- 30, 2014

Indo Global Journal of Pharmaceutical Sciences( ISSN 22491023 ; CODEN- IGJPAI; NLM ID: 101610675) indexed and abstracted in EMBASE(Elsevier), SCIRUS(Elsevier),CABI, CAB Abstracts, Chemical Abstract Services(CAS), American Chemical Society(ACS), Index Copernicus, EBSCO, DOAJ, Google Scholar and many more. For further details, visit 